\title{
New classification of spinal lipomas based on embryonic stage
}

\author{
Nobuhito Morota, MD, ${ }^{1}$ Satoshi Ihara, MD, ${ }^{1}$ and Hideki Ogiwara, MD, PhD² \\ 1Division of Neurosurgery, Tokyo Metropolitan Children's Medical Center; and 2Division of Neurosurgery, National Center for \\ Child Health and Development, Tokyo, Japan
}

\begin{abstract}
OBJECTIVE Spinal lipomas are generally thought to occur as a result of failed primary neurulation. However, some clinical features cannot be explained by this theory. The authors propose a novel classification of spinal lipomas based on embryonic changes seen during primary and secondary neurulation.
\end{abstract}

\begin{abstract}
METHODS A total of 677 patients with occult spinal dysraphism underwent 699 surgeries between August 2002 and May 2015 at the National Center for Child Health and Development and Tokyo Metropolitan Children's Medical Center. This group of patients had 378 spinal lipomas, including 119 conus spinal lipomas, 27 lipomyelomeningoceles, and 232 filum lipomas, which the authors classified into 4 types based on neural tube formation during embryonic development. Type 1 is defined as pure primary neurulation failure; Type 2 ranges from primary to secondary neurulation failure; Type 3 consists of secondary neurulation failure (early phase); and Type 4 is defined as secondary neurulation failure (late phase). The authors also review embryogenesis in secondary neurulation and analyze the clinical utility of the new classification.
\end{abstract}

RESULTS There were 55 Type 1 spinal lipomas, 29 Type 2, 62 Type 3, and 232 Type 4. All filum lipomas fell into the Type 4 spinal lipoma category. Association with anorectal and/or sacral anomalies was seen in none of the Type 1 cases, $15(52 \%)$ of Type 2, $35(56 \%)$ of Type 3, and $31(13 \%)$ of Type 4. Urogenital anomalies were observed in none of the Type 1 or Type 2 cases, $1(2 \%)$ of Type 3, and 28 (12\%) of Type 4 . Anomaly syndromes were present in none of the Type 1 cases, $6(21 \%)$ of Type 2, $3(5 \%)$ of Type 3, and $16(7 \%)$ of Type 4 . Associated anomalies or anomaly syndromes were clearly observed only for Type $2-4$ spinal lipomas encompassing failed secondary neurulation. Radical resection was feasible for Type 1 spinal lipomas.

CONCLUSIONS Secondary neurulation of the spinal cord gives rise to the conus medullaris and filum terminale, which are often involved in spinal lipomas. Formation of spinal lipomas seems to be a continuous process overlapping primary and secondary neurulation in some cases. Association with other anomalies was higher in Type 2-4 spinal lipomas, which included failed secondary neurulation, than in Type 1 lipomas, with failed primary neurulation. On the other hand, radical resection was indicated for Type 1, but not for Type 2, spinal lipomas. The new classification of spinal lipomas based on embryonic stage has the potential for clinical use and agrees well with both clinical and surgical findings. The classification proposed here is still preliminary. Further studies and verification are necessary to establish its clinical utility.

https://thejns.org/doi/abs/10.3171/2016.10.PEDS16247

KEY WORDS spinal lipoma; classification; embryology; primary neurulation; secondary neurulation; spine

$\mathrm{T}$ HE human spinal cord is formed following the primary and secondary neurulations. Failed neurulation leads to various types of spinal developmental malformations. Although primary neurulation is relatively well understood, less is known about the process of secondary neurulation or its clinical importance.

Spinal lipomas are among the most common spinal developmental malformations encountered in pediatric neurosurgery, representing a group of disorders known as occult spinal dysraphism, which is often associated with "pathological" spina bifida. ${ }^{8}$ The embryonic background of spinal lipoma cases is marked by a premature disjunction between the neural and cutaneous ectoderm during the process of primary neurulation. ${ }^{14}$ Failed primary neurulation allows invasion of mesenchymal tissue of mesodermic origin into the neural structure, leading to the formation of a spinal lipoma. An unfused spinal cord caused by failed primary neurulation remains dysraphic in spinal lipoma.

Despite most spinal lipomas being regarded as the result of failed primary neurulation, some confusion in terms of 
the embryogenesis of spinal lipomas remains. Embryologists often consider closed spinal lesions to be the result of failed secondary neurulation. ${ }^{3}$ Indeed, the presence of a lipoma in the filum terminale, the final product of secondary neurulation, is increasingly being acknowledged. The availability of MRI has made the discovery of caudally located lipomas, which do not penetrate the dorsal aspect of the dura mater or fascia, and are unassociated with spina bifida, more common. It should be remembered that spina bifida diagnosed in such patients does not constitute true "pathological" spina bifida, but merely "physiological spina bifida" formed by the cartilaginous part of the lamina in the course of normal development. ${ }^{28}$

Surgically orientated classification of spinal lipoma was first proposed by Chapman and underwent various modifications based on MRI findings. $1,2,8,16,18$ Chapman's classification is still useful today but does not account for spinal lipomas originating from failed secondary neurulation. Some have included the filum lipoma in the classification. ${ }^{1}$ However, because this classification is simply based on the site and morphology of the spinal lipoma, it is now considered to have less clinical significance.

The purpose of this paper is to classify spinal lipomas based on caudal spinal cord formation during embryogenesis. The formation of spinal lipomas from primary to secondary neurulation was considered, and the conditions were classified based on the stage of embryogenesis. The implications and clinical relevance to associated malformations were also assessed from a surgical point of view.

\section{Methods}

A total of 677 patients with occult spinal dysraphism underwent 699 surgeries between August 2002 and May 2015 at the National Center for Child Health and Development and Tokyo Metropolitan Children's Medical Center. In this group of patients, there were 119 conus spinal lipomas (including dorsal, caudal, and transitional types), 27 lipomyelomeningoceles (defined as "the terminal spinal cord herniated outside the confines of the spinal canal with the subarachnoid space"8), and 232 filum lipomas. In addition, there were 214 cases of thickened filum terminale and 85 instances of other occult spinal dysraphic pathologies (dermal sinus tract, split cord malformation, limited dorsal myeloschisis, etc.), although these were excluded from the study. Data were retrospectively collected for the total of 378 spinal lipomas. This study was approved by the institutional review board of Tokyo Metropolitan Children's Medical Center.

\section{Spinal Lipoma Classification}

The diagnosis and classification of spinal lipomas were made on the basis of T1- and T2-weighted MRI using mid- and parasagittal views. Axial MR images offered useful information for resection of spinal lipomas but not for their classification. Spinal CT scans were performed in all patients. Reconstructed 3D images of the lumbosacral spine and pelvis were used to diagnose pathological spina bifida and sacrococcygeal bony anomalies.

The spinal lipomas in this study were classified by the senior author (N.M.) and by one of the coauthors (S.I. and
H.O.), both of whom are experienced pediatric neurosurgeons. All of the lipomas were classified using both the conventional classification system and the new system proposed in the present paper. The new classification of spinal lipomas is based on the stages of neural tube formation during embryogenesis. The individual types of spinal lipoma are defined below and in Fig. 1.

Conus spinal lipomas, lipomyelomeningoceles, and filar lipomas were reclassified according to the new classification system. The presence of anorectal and/or sacral anomalies (anal atresia, sacral hypogenesis, and so on), urogenital anomalies (hypospadias, uterus bicornis, and so on), double lipomas, and associated anomaly syndromes was analyzed. Radical resection of the spinal lipoma, first introduced in April 2009 in this series, was also analyzed using the new classification. ${ }^{16,18,19}$

\section{New Classification System Used in this Study}

Type 1: Primary Neurulation Failure Only

Type 1 spinal lipoma is a typical form in which the lipoma-cord interface occurs on the dorsal surface of the spinal cord, while the conus medullaris is located distally or ventrally with respect to the caudal end of the lipoma. The subcutaneous fat mass penetrates the fascia and reaches the dorsal surface of the dura through the pathological spina bifida. ${ }^{28}$ The intradural lipoma is usually located in the dorsal or dorsolateral aspect of the spinal cord, which is tethered caudally (Fig. 2).

\section{Type 2: Failed Neurulation Between the Primary and Secondary Stages}

Type 2 spinal lipoma also penetrates the fascia and continues into the intraspinal lesion through the pathological spina bifida. The lipoma is attached to the spinal cord, but the conus medullaris is malformed and difficult to recognize by MRI (Fig. 3).

The difference between Types 1 and 2 is determined by the presence or absence of the conus medullaris. Because Type 1 spinal lipomas are formed purely by failed primary neurulation, the conus medullaris is present caudally or ventrally with respect to the spinal lipoma. The tapered shape of the end of the spinal cord suggests the presence of the conus medullaris (Fig. 4). On the other hand, the Type 2 spinal lipoma is the result of failed neurulation between the primary and secondary stages. The end of the spinal cord where the conus medullaris should exist is undifferentiated and merges with the spinal lipoma. A sharp tapered end to the spinal cord is difficult to confirm in Type 2 spinal lipomas (Fig. 5).

\section{Type 3: Early Phase Secondary Neurulation Failure}

This type of spinal lipoma involves the conus medullaris, the caudal end of which is unformed and directly connects to the spinal lipoma. The lipoma extends in the caudal direction, penetrating the caudal end of the dura to connect with subcutaneous fat through the enlarged sacral hiatus. The dorsal surface of the dura remains intact. In contrast to Type 2, in Type 3 spinal lipomas there is no pathological spina bifida or fascial defect due to the lipoma (Fig. 6). 


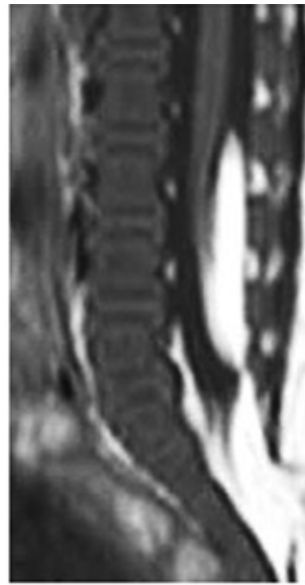

Type 1

Primary neurulation failure

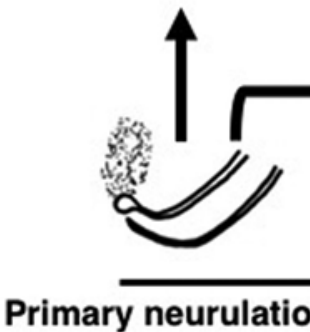

Primary neurulation

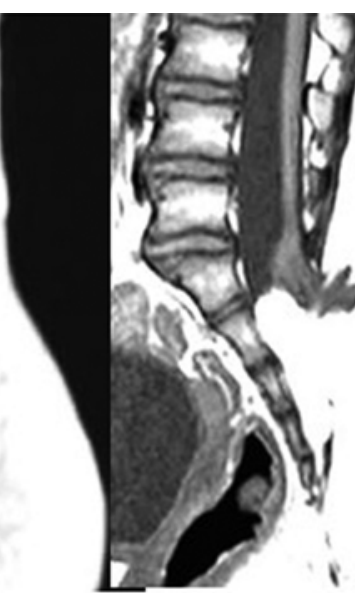

Type 2

Primary/secondary (junctional)

neurulation failure

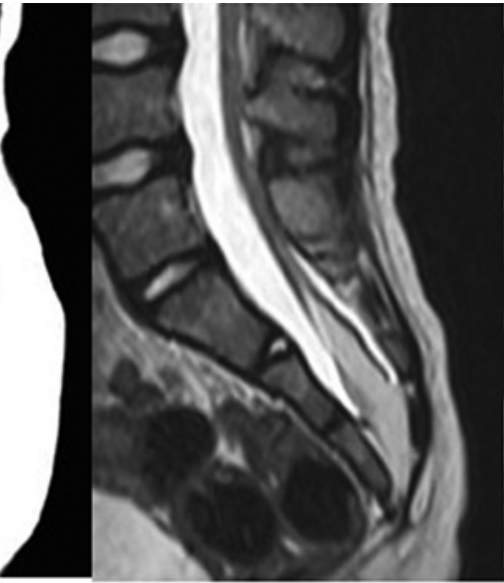

Type 3

Secondary neurulation Secondary neurulation failure (early phase)

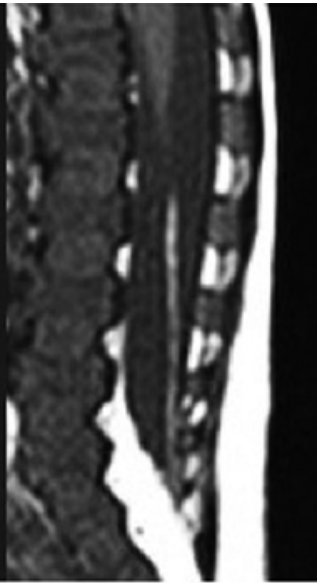

failure (late phase)

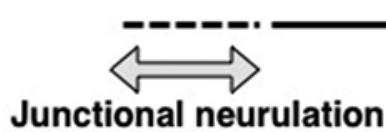

Secondary neurulation

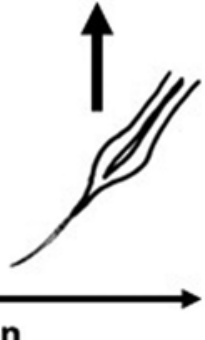

FIG. 1. The proposed new classification of spinal lipomas and their embryonic background are shown. Failure of primary neurulation results in Type 1 spinal lipoma. Type 2 spinal lipoma is the product of failed neurulation between the primary and secondary stages including junctional neurulation. Type 3 spinal lipoma results from failed early phase secondary neurulation, while Type 4 results from a failure in the late phase.

Type 4: Late Phase Secondary Neurulation Failure

In this type, the spinal lipoma is located in the filum terminale. The caudal end of the conus medullaris is easily confirmed on MRI, and most or part of the filum terminale is composed of a linear string-like lipoma.

\section{Statistical Analysis}

Data on associated anomalies, anomaly syndromes, and double lipomas were analyzed statistically for the different types of spinal lipoma and compared. A chi-square test was first applied to detect any difference. If a difference was detected, Bonferroni correction was introduced to adjust the confidence intervals between these cohorts. A $p$ value less than 0.05 was considered statistically significant. All statistical analyses were conducted using IBM SPSS Statistics version 23 (IBM Corp.).

\section{Results}

Of the previously diagnosed 119 cases of conus lipomas, 49 were newly classified as Type 1, 14 as Type 2, and 56 as Type 3 (Table 1). Anorectal and/or sacral anomalies were confirmed clinically or by CT images in $10(71 \%)$ of the patients with Type 2 lipomas, $33(59 \%)$ of the patients (59\%) with Type 3 lipomas, and none of the patients with a
Type 1 lipoma. A urogenital anomaly was reported in only 1 patient with a Type 3 lipoma. A double lipoma, ${ }^{26}$ another form usually located in the filum terminale, was observed in $1(2 \%)$ of the cases of Type $1,11(79 \%)$ of the cases of Type 2, and $3(5 \%)$ of the cases of Type 3 lipomas. Regarding associated conditions, extrophy of the cloaca was present in $4(29 \%)$ of the patients with a Type 2 lipoma, and VACTER syndrome was present in $3(5 \%)$ of the patients with a Type 3 lipoma. None of the patients with a Type 1 lipoma showed evidence of anomaly syndromes (Table 2). Of the 69 total cases surgically treated after April 2009, radical or semiradical resection of the spinal lipoma was carried out in 12 cases of Type 1 lipomas, 1 case of Type 3 lipoma, and no case of Type 2 lipoma.

Of the 27 patients with lipomyelomeningocele, 6 had a Type 1, 15 had a Type 2, and 6 had a Type 3 lipoma (Table 1). Associated anorectal and/or sacral anomalies were diagnosed in $5(33 \%)$ of the patients with a Type 2, 2 (33\%) of the patients with a Type 3 , and none of the patients with a Type 1 lipoma. No urogenital anomalies were observed in this group. Double lipomas were present in $5(33 \%)$ of the patients with a Type 2 and none of the patients with a Type 1 or 3 lipoma. Extrophy of the cloaca was present in $2(13 \%)$ of the patients with a Type 2 lipoma. None of the patients with a Type 1 or 3 lipoma had any association 

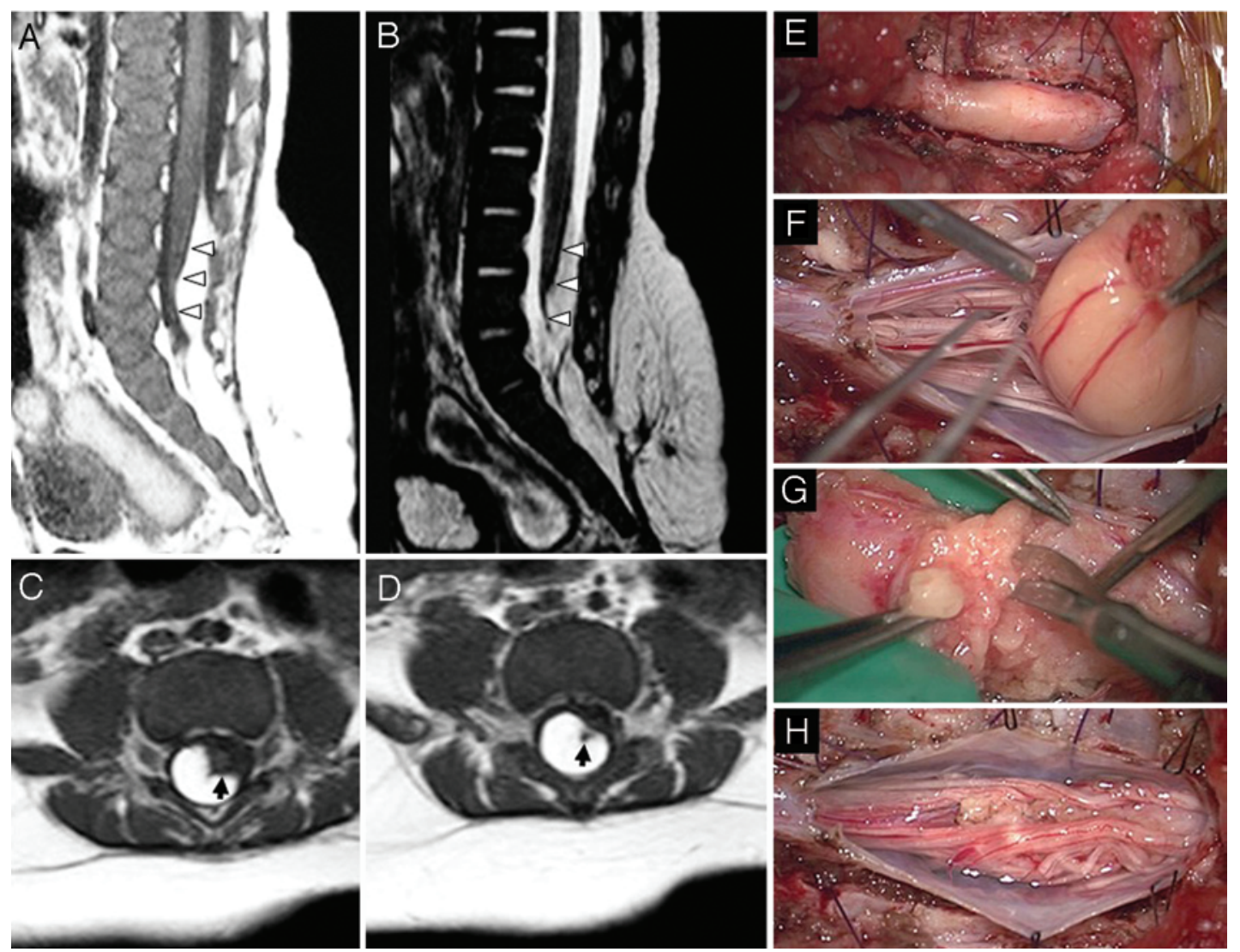

FIG. 2. A case of Type 1 spinal lipoma. This 3-month-old girl was born with a hump on her back. MRI revealed a spinal lipoma. The lipoma was visible dorsal to the conus medullaris in the sagittal T1- and T2-weighted images (A and B, respectively). The lipoma extended caudally and connected to the subcutaneous fat through the spina bifida. The borderline (white triangles) between the lipoma and conus medullaris, which preserved its cone shape, was smooth. Axial T1-weighted MR images (C and D) showed the conus medullaris (arrow) covered with the dorsal to dorsolaterally extended lipoma. The lipoma resembled the transitional type but was judged Type 1 because the conus medullaris was present ventral to the lipoma. Intraoperatively, the spinal lipoma could be observed merging with the subcutaneous fat after laminectomy $(E)$. The dura was opened, and the lipoma was untethered from the dura. The caudal end of the lipoma was reflected to dissect the cauda equina to the point where the tip of the conus medullaris was located (F). The lipoma was radically dissected from the rostral side $(\mathbf{G})$. The photograph in $\mathbf{H}$ shows the surgical field after removal of the lipoma and myeloplasty before dural closure. In all of the photographs, right is rostral and left is caudal. Figure is available in color online only.

with anomaly syndromes (Table 2). Radical and semiradical resection of the spinal lipoma was contraindicated for all patients with lipomyelomeningocele, regardless of type.

All filum lipoma cases were categorized as Type 4. Anorectal and/or sacral anomalies were observed in 31 patients (13\%) and urogenital anomalies in $28(12 \%)$. Two children with duplicated filum terminale had a filum lipoma in each of the 2 terminal fila (i.e., double filum lipomas). ${ }^{21}$ Extrophy of the cloaca was found in $3(1 \%)$, VACTER syndrome in $12(5 \%)$, and Currarino syndrome in $1(0.4 \%)$ of the patients (Table 2$)$.

Special attention was paid to the relationship between the new classification and associated anomalies. Of the 378 spinal lipomas, 55 were Type 1 (patient age range 14 days25 years, median 4 months), 29 were Type 2 (patient age range 1 month-22 years, median 4 months), 62 were Type 3 (patient age range 12 days -23 years, median 3 years), and 232 were Type 4 (patient age range 2 months-29 years, median 1 year). There was no association with anorectal and/ or sacral anomalies in any of the Type 1 cases; associated anorectal or sacral anomalies were present in 15 (52\%) of the Type 2 cases, 35 (56\%) of the Type 3 cases, and 31 (13\%) of the Type 4 cases. After Bonferroni correction, Type 2 and Type 3 spinal lipomas showed a significantly stronger association with anorectal and/or sacral anomalies than Type 1 or Type 4 ( $p<0.001)$. There was a weak and statistically nonsignificant association with anorectal and/or sacral anomalies for Type 1 and Type $4(p=0.06)$. There was no statistically significant difference between Type 2 and Type 3 with respect to association with these anomalies. Urogenital anomalies were observed in none of the patients with Type 1 or Type 2 spinal lipomas, 1 (2\%) of those with Type 3 , and 28 (12\%) of those with Type 4. A statistically significant difference with respect to association with urogenital anomalies was seen only between Type 1 and Type 4 spinal lipoma $(p=0024)$. A weak but statistically nonsignificant association with urogenital anomalies was detected for Types 3 and $4(p=0.084)$. No association was present for Types 2 and 4, probably due to the small number of patients with Type 2 spinal lipomas. Anomaly syndromes were present in none of the patients with Type 1 lipomas, 6 (21\%) of those with Type 2,3 (5\%) of those with 


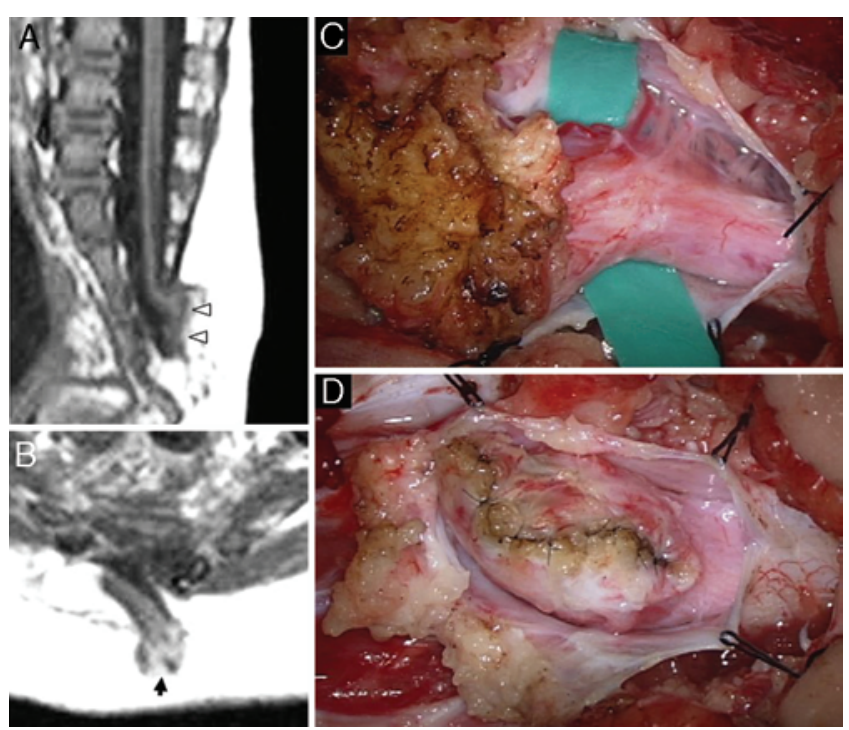

FIG. 3. A case of Type 2 spinal lipoma. This 6 -month-old girl was born with a hump on her back. MRI revealed a spinal lipoma (A and B, respectively, sagittal and axial T1-weighted images). Sagittal images showed the spinal cord and subarachnoid space extruding from the spinal canal. The conus medullaris was unformed, and the caudal end of the spinal cord was connected unevenly with the subcutaneous fat (arrowheads). Axial images demonstrated the extruded spinal cord merging with subcutaneous fat (arrow). The lipoma was judged as Type 2 because the conus medullaris was incomplete. The intraoperative photograph in $\mathbf{C}$ shows the spinal cord and lipoma after untethering. Note that the enlargement of the end of the spinal cord and its extension into the lipoma. The lipoma was partially removed and myeloplasty was performed (D). Figure is available in color online only.

Type 3, and 16 (7\%) of those with Type 4. A statistically significant difference with respect to the association with anomaly syndromes was confirmed only for Types 1 and 2 spinal lipoma $(\mathrm{p}=0.006)$. A double lipoma was found in 1 (2\%) of the patients with Type 1 spinal lipomas, $16(55 \%)$ of those with Type 2, $3(5 \%)$ of those with Type 3, and 2 (1\%) of those with Type 4 . Type 2 spinal lipoma showed a significantly higher association with double lipoma than Types 1,3 , and $4(\mathrm{p}<0.001)$. It is interesting to note that associated anomalies or anomaly syndromes were clearly observable only in Types 2, 3, and 4, all of which involved failed secondary neurulation (Table 2).

\section{Discussion}

\section{Embryonic Process of Neural Tube Formation}

Details of the mechanism and process of caudal neural tube formation have become known only during the past few decades (Fig. 7). Primary neurulation, announced by the appearance of the neural groove and folds, starts after about $17-18$ postovulatory days (PODs) in the normal human embryo. ${ }^{13}$ Fusion of the neural folds begins approximately after POD 22. Initiation of neural tube fusion is believed to start at multiple sites simultaneously with bi- or unidirectional extension. ${ }^{3,27}$ The caudal end of the neuropore starts closing approximately after POD 26 . It is well known that the site of the final closure of the caudal end corresponds to the S-2 vertebral level. ${ }^{13}$ During the process of primary neurulation, separation of the cutaneous ectoderm from the neuroectoderm, referred to as a "dys- junction," develops after neural tube fusion. ${ }^{8,15}$ Premature dysjunction (i.e., dysjunction preceding neural fold fusion and allowing invasion of mesodermal tissues into the neural tube) leads to the development of the spinal lipoma. ${ }^{8,15}$

Secondary neurulation-which begins at POD 26-27, when the caudal neuropore closes ${ }^{10}$ - differs from primary neurulation in that it neither involves the ectoderm nor forms a neural canal from the neural plate. Instead, secondary neurulation is the process in which mesenchymal cells undergo epithelialization and tubulogenesis. ${ }^{25}$ As a prelude to this, pluripotent tissues of the caudal eminence (tail bud), consisting of neural and mesodermal tissues, appear distal to the caudal neuropore at POD 20-22. The caudal eminence gives rise to the caudal portion of the digestive tube, blood vessels, and the future somites of the S1-2 vertebral level and below-and eventually the spinal cord..$^{10-13}$ The caudal eminence fuses with the caudal neuropore and forms a solid cellular mass called the neural cord at POD 26 , which then undergoes cavitation and connects to the central canal already present in the neural tube. By this process of cavitation, the solid neural cord transforms into a hollow secondary neural tube and fuses with the neural tube formed by primary neurulation into a continuous entity. ${ }^{3,13}$ The hollow secondary neural tube starts to regress after POD 28 to 32, and partly retreats to the fibrous layer, which eventually degenerates into the filum terminale. ${ }^{12}$ This process of degeneration and differentiation is called "retrogressive differentiation.", 12 It is estimated that secondary neurulation continues until at least postovulatory Week $7 .{ }^{13}$ In short, secondary neurulation occurs in 4 steps. The first is the condensation and formation of the caudal eminence; the second is the cavitation of the caudal eminence and connection to the closed primary neural tube (spinal cord); the third consists of the fusion of the hollow secondary neural tube and the primary neural tube; and the fourth is the regression of the secondary neural tube to the filum terminale (Fig. 7).

The transition from primary to secondary neurulation proceeds seamlessly, but the detailed mechanism is not yet fully understood. Formation of the caudal neural tube comprises 3 developmental stages. Closure of the caudal neuropore occurs first, followed by secondary neurulation and retrogressive degeneration of the secondary neural tube. ${ }^{12}$

Recent studies suggest the presence of a unique process called "junctional neurulation," bridging the primary and secondary neurulations. ${ }^{4}$ Presence of the junctional region between the primary and secondary neurulation has also been reported. ${ }^{24}$ The complex process of caudal neural tube formation is also implicated in the development of various types of spinal lipomas. It should be borne in mind, however, that the process of neurulation described here is mainly based on animal models, and the human process could be different.

\section{Formation of Anorectal and Urogenital Organs}

Knowledge of the basic embryology of the anorectal and urogenital organs is crucial to understanding the close relationship these organs have to secondary neurulation. These organs are not only located in close proximity to the caudal end of the spinal cord but also develop simultaneously with secondary neurulation. 

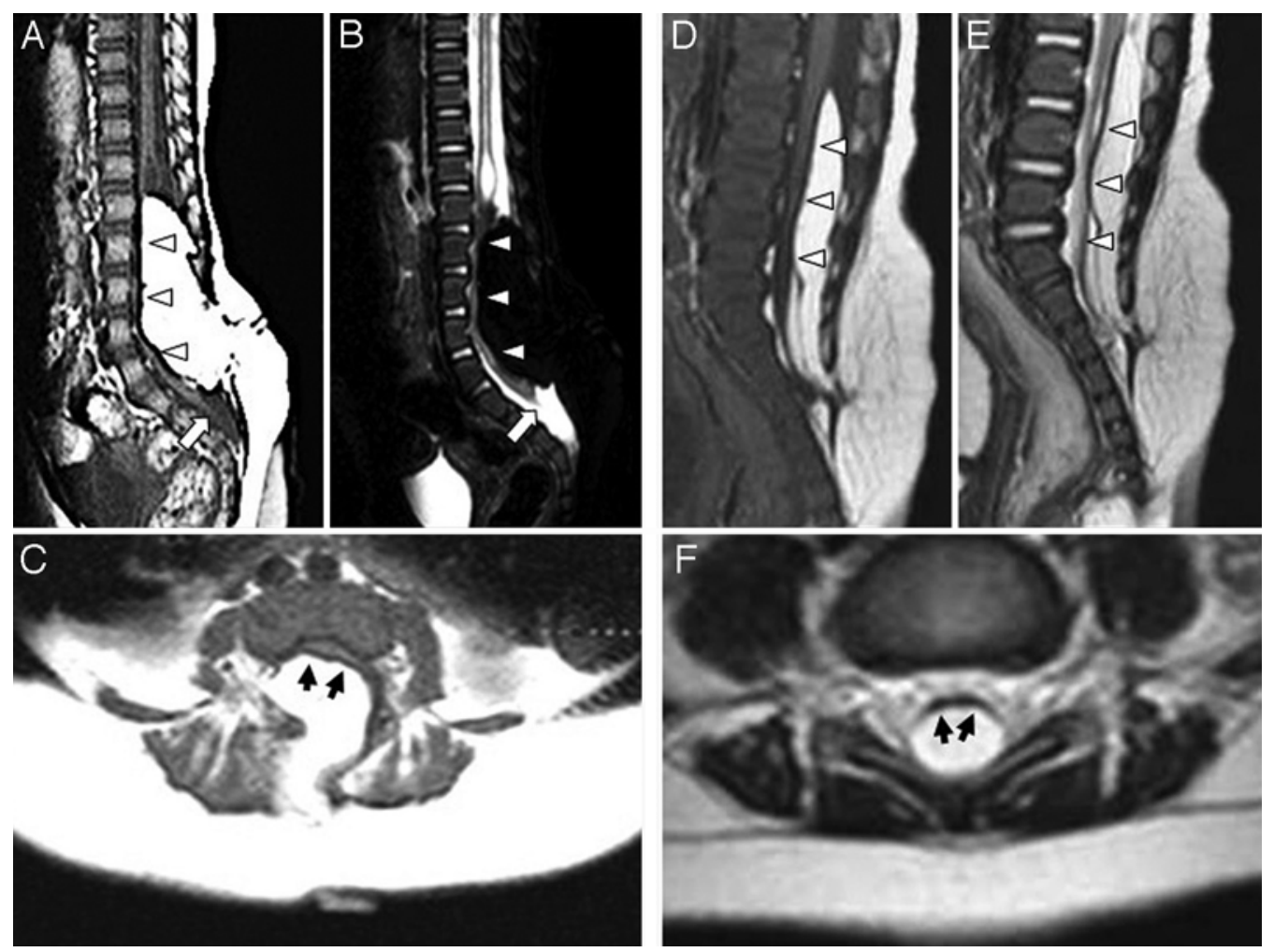

FIG. 4. MR images of Type 1 spinal lipomas. A-C: Sagittal T1-weighted (A) and T2-weighted (B) images showing a huge spinal lipoma compressing the spinal cord ventrally with relatively smooth borderline (arrowheads) and axial T1-weighted image (C) showing a flattened spinal cord with smooth borderline (black arrows). Note in the sagittal images that the tip of the conus medullaris is caudal to the spinal lipoma (white arrows). D-F: Sagittal T1-weighted (D) and T2-weighted (E) images showing a spinal lipoma located dorsally with respect to the caudal end of the spinal cord, and axial T1-weighted image (F) showing a flattened spinal cord with smooth borderline (black arrows). Note in the sagittal images that the conus medullaris is ventral to the spinal lipoma but the cone shape is preserved.

The cloaca, the distal end of the fetal digestive tube, is connected to the hindgut posteriorly and to the allantois anteriorly. The urorectal septum extends caudally between them from the end of postovulatory Week 5, reaching the cloacal membrane at postovulatory Week 8 , thus separating the cloaca from the rectum and anal membrane posteriorly and from the urogenital sinus and membrane anteriorly.22 Organogenesis proceeds thereafter. Formation of external genitalia begins at postovulatory Week 7, after separation of the anal and urethral folds (Fig. 7) ${ }^{23}$ It should be remembered that the caudal eminence is located dorsal to the cloaca; the chronological and anatomical coincidence with secondary neurulation can lead to interactions resulting in complex anomalies such as caudal agenesis. ${ }^{14}$

\section{Classification of Spinal Lipomas}

Classification of spinal lipomas has historically been based on the morphology of lipomas in relation to the spinal cord. Chapman, ${ }^{2}$ considering the site of lipoma attachment to the cord and its anatomical relationship to the posterior spinal nerve root, classified spinal lipomas into 3 types, the dorsal, caudal, and transitional. His historical classification, based on his surgical observations before the advent of MRI, still remains fundamental and has been modified to form the basis of various other classificatory systems. To this classification, Arai et al. ${ }^{1}$ added the filar lipoma, based on MRI findings, and the lipomyelomeningocele, which may sometimes present the intrusion of a subcutaneous lipoma through a defect in the fascia into the central canal. McLone and Thompson advocated classifying the spinal lipoma into those with intact dura (subpial lipomas), those of the conus medullaris, and those of the filum terminale. ${ }^{8}$ They further subdivided the lipomas of the conus medullaris into 3 groups, with the lipomyelocele exemplifying spinal lipomas resulting from failed primary neurulation.

Pang first introduced the embryological perspective into the classification of spinal lipomas by proposing the dorsal, terminal, and transitional lipomas types, ${ }^{15}$ with lipomyelomeningoceles being included in the latter. The dorsal and transitional types develop during primary neurulation, whereas the terminal type develops in secondary neurulation. Pang later added the chaotic lipoma associated with the embryological stage between the transitional and terminal lipomas..$^{18,20}$

The difference between the previous and new classifications is shown in Fig. 8. The Type 1 spinal lipoma includes previously classified transitional lipomas if the conus medullaris is present ventrally despite compression of the lipoma, which is located dorsally and extends caudally. Pang conflated the caudal and filar lipomas with the terminal lipoma because they were the products of failed 


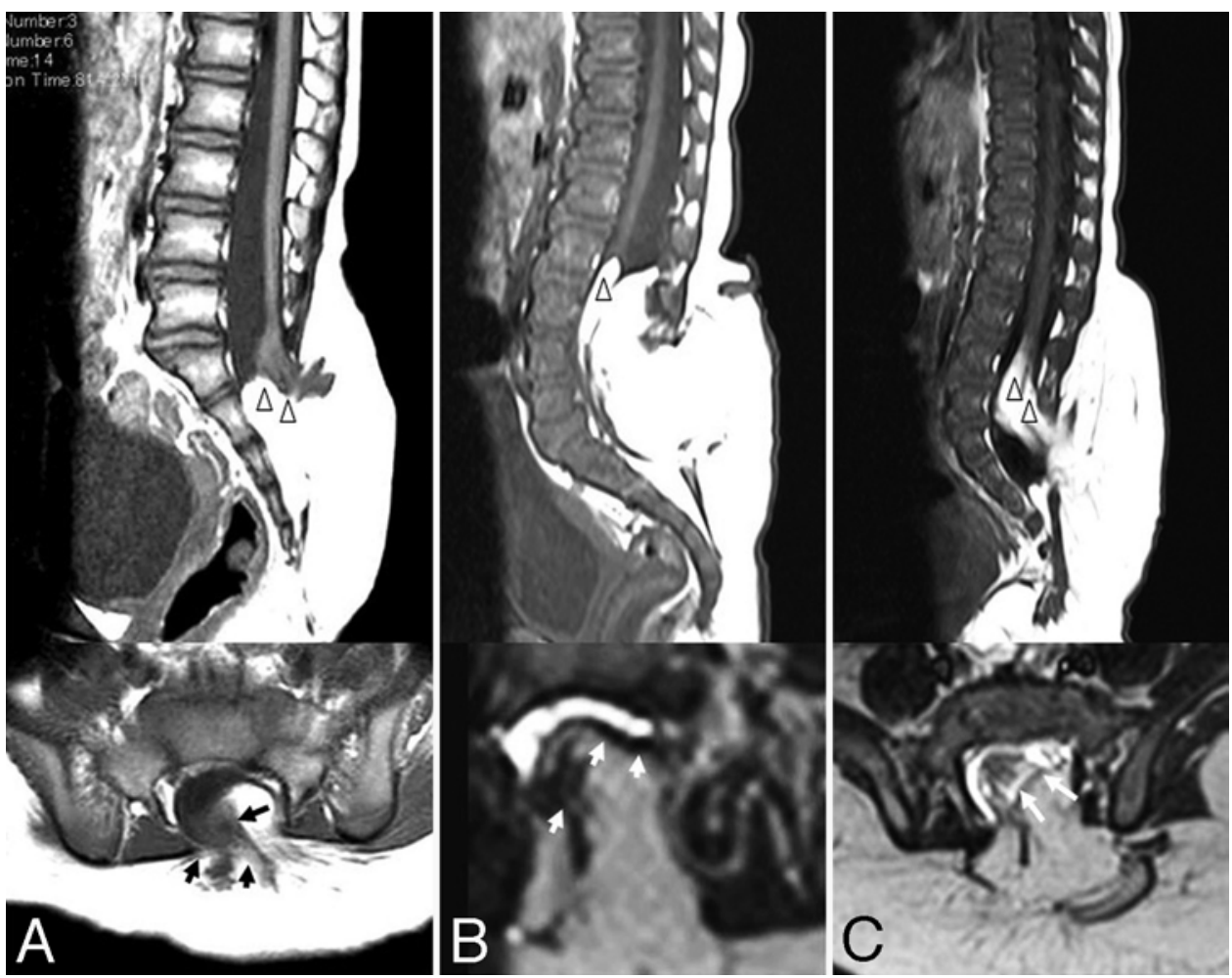

FIG. 5. MR images of Type 2 spinal lipomas. A: Sagittal (upper) and axial (lower) T1-weighted images. The caudal end of the spinal cord is enlarged and disrupted without forming the conus medullaris (arrowheads). A part of neural tissue extrudes from the spinal canal. The T1-weighted axial image demonstrates the spinal cord merged with irregularly shaped subcutaneous fat (black arrows). B: Sagittal T1-weighted (upper) and axial T2-weighted (lower) images. The spinal cord merges with the spinal lipoma without forming the conus medullaris (arrowhead). The T2-weighted axial image reveals the irregular shape of the borderline with neural components protruding into the subcutaneous fat (white arrows). C: Sagittal T1-weighted (upper) and axial T2-weighted (lower) images. The spinal cord connects with the spinal lipoma without a clear borderline (arrowheads), and the conus medullaris is not present. The T2-weighted axial image demonstrates the spinal cord fused to the lipoma with indistinct margins (white arrows).

secondary neurulation. ${ }^{14}$ In the new classification, terminal lipomas were once again categorized into separate types, Types 3 and 4, based on the early or late phase of secondary neurulation. If the lipoma originates directly from the terminal region of the conus medullaris, it is judged to be a Type 3 spinal lipoma. In Type 4 spinal lipomas, the normal end of the conus medullaris is present and moves toward the filum terminale. This then positions the lipoma at the caudal side of the filum terminale.

\section{Embryonic Stages and the New Classification of Spinal Lipomas}

Type 1 spinal lipomas are the result of premature dysjunction between the neuro- and cutaneous ectoderm. (Refer to Fig. 1 for an overview of the 4 types.) Premature dysjunction seems widely considered by pediatric neurosurgeons to be the mechanism of spinal lipoma development. ${ }^{8,15,20}$ It is clear that premature dysjunction is the failure of primary neurulation, which leads to Type 1 spinal lipomas. It also explains the morphological features of Type 1 spinal lipomas, which attach and compress the spinal cord from the dorsal or dorsolateral site in association with pathological spina bifida..$^{28}$

Type 4 spinal lipomas, usually known as filum lipomas, are generated by a different mechanism from that seen in
Type 1 . While previous classifications placed the filum lipoma together with other lipomas, ${ }^{1,8}$ Pang considered it to be a form of terminal lipoma and distinct from the caudal type. ${ }^{15,20}$ Pang described the terminal lipoma as the product of abnormal secondary neurulation, pointing out, however, the possibility of the involvement of both primary and secondary neurulation in its formation. Type 4 spinal lipomas are apparently equivalent to what he calls "incomplete or ineffective degeneration phase at late secondary neurulation, possibly due to failed apoptosis." 20

Type 3 spinal lipoma corresponds to the caudal type of lipoma in the conventional classification, ${ }^{1,2,8}$ or to another type of terminal lipoma in Pang's classification, which develops early in secondary neurulation around the time of the condensation of the caudal eminence. ${ }^{20}$ Type 3 spinal lipomas have no pathological spinal bifida or fascial defect caused by penetrating fatty tissue. ${ }^{28}$ They attach in various ways to the conus medullaris but always distal to the last branch of the spinal nerves..$^{15,20}$

Type 2 spinal lipomas are thought to result from failed junctional neurulation; the period during which Type 2 spinal lipomas develop extends from primary to secondary neurulation, thus explaining why Type 2 spinal lipomas do not follow the developmental sequence observed in Type $1 .^{20}$ Type 2 spinal lipomas include some forms of transitional lipoma, the lipomyelomeningocele, and the 


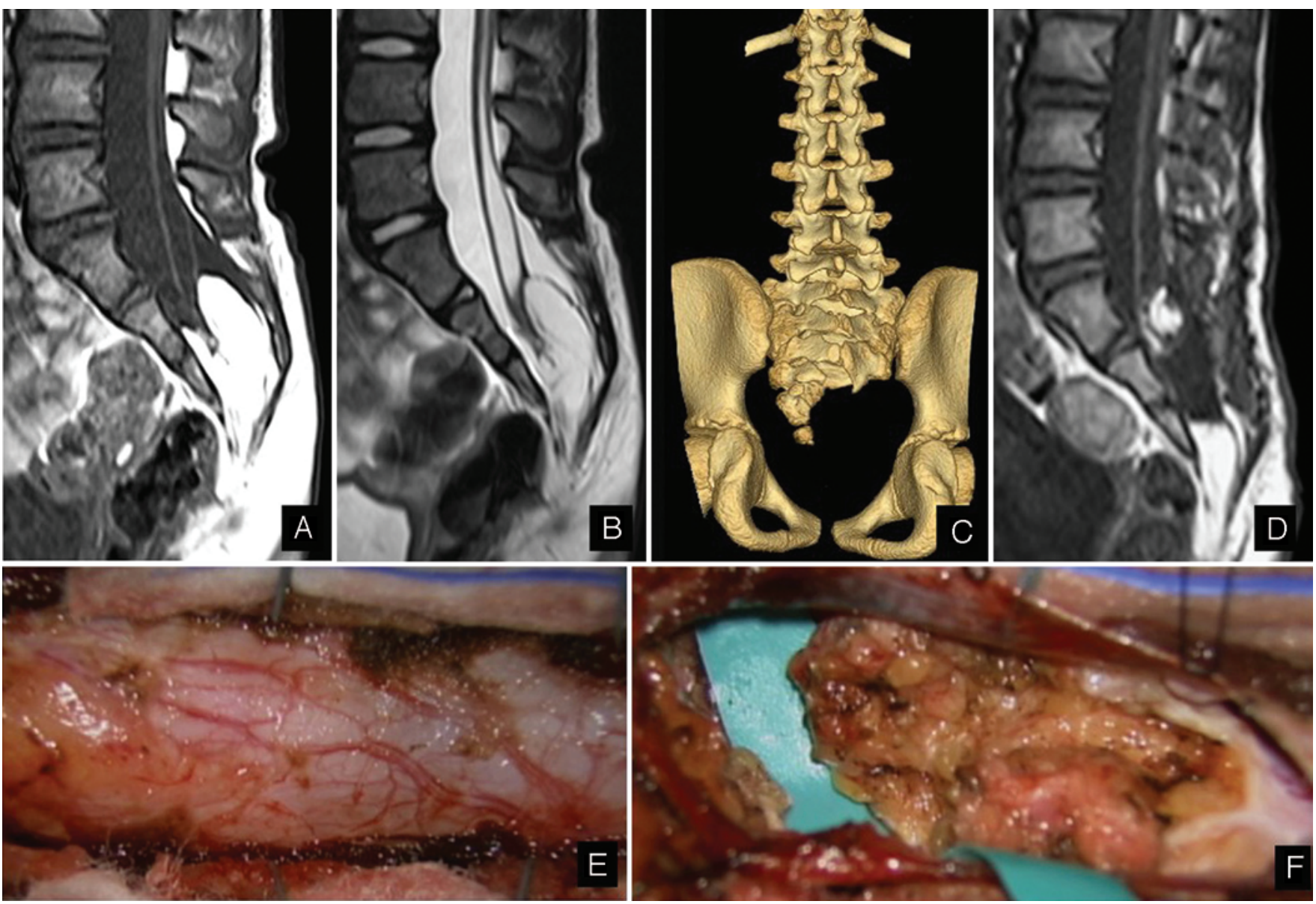

FIG. 6. A case of Type 3 spinal lipoma. This 7-year-old girl had neurogenic bladder, and MRI revealed a spinal lipoma. There was no skin stigma on her back. Sagittal T1-weighted (A) and T2-weighted (B) MR images revealed a lipoma dorsal to the caudal aspect of the conus medullaris with syringomyelia. The lipoma resembled the transitional type of conus lipoma but was judged to be Type 3 because it extended caudally without penetrating the dorsal dura or fascia, passed the sacral hiatus, and merged with subcutaneous fat. Right sacral hypogenesis was present, as can be seen in the 3D reconstructed CT image (C). The patient underwent partial resection of the lipoma and untethering of the spinal cord (postoperative T1-weighted MR image, D). No dorsal dural defect was seen intraoperatively, although an intradural lipoma was observed through the dura on the caudal side (E, left). The photograph in $\mathbf{F}$ was obtained after partial resection of the lipoma and untethering the spinal cord but before myeloplasty. The lipoma was fused with the conus medullaris obliquely. No clear fusion line was confirmed. The cutting line was drawn distal to the caudalmost nerve roots. In both photographs right is rostral and left is caudal. Figure is available in color online only.

chaotic lipoma. Because developmental failure begins at primary neurulation, Type 2 spinal lipomas are associated with pathological spina bifida and attach to the dorsal side of the spinal cord. Due to the involvement of junctional neurulation, condensation of the caudal eminence and its fusion to the neural tube are disturbed, and the lipoma ex-

TABLE 1. Classification of spinal lipomas

\begin{tabular}{cccc}
\hline Characteristic & Conus Lipoma & Lipomyelomeningocele & Filum Lipoma \\
\hline No. of patients & 119 & 27 & 232 \\
\hline Age at surgery & & & 1 yr \\
\hline Median & 9 mos & 4 mos & 2 mos-29 yrs \\
\hline Range & 27 days-25 yrs & & 0 \\
\hline Classification of lipoma & & $6(22 \%)$ & 0 \\
\hline Type 1 & $49(41 \%)$ & $15(56 \%)$ & 0 \\
\hline Type 2 & $14(12 \%)$ & $6(22 \%)$ & $232(100 \%)$ \\
\hline Type 3 & $56(47 \%)$ & 0 & 0 \\
\hline Type 4 & 0 & & \\
\hline
\end{tabular}

Values represent numbers of patients (\%) unless otherwise indicated. 
TABLE 2. Associated anomalies

\begin{tabular}{lcccc}
\hline \multirow{2}{*}{ Characteristic } & \multicolumn{4}{c}{ Spinal Lipoma Type } \\
\cline { 2 - 5 } & Type 1 & Type 2 & Type 3 & Type 4 \\
\hline No. of patients & 55 & 29 & 62 & 232 \\
\hline Age at surgery & & & 3 yrs & 1 yr \\
\hline Median & 4 mos & 4 mos & 12 days-23 yrs & 2 mos-29 yrs \\
\hline Range & 14 days-25 yrs & 1 mo-22 yrs & & $31(13 \%)$ \\
\hline Associated anomalies & & & $35(56 \%)$ & $28(12 \%)$ \\
\hline Anorectal/sacral anomalies & 0 & $15(52 \%)$ & $1(2 \%)$ & $16(7 \%)$ \\
\hline$\quad$ Urogenital anomalies & 0 & 0 & $3(5 \%)$ & $2(1 \%)$ \\
\hline Anomaly syndromes & 0 & $6(21 \%)$ & $3(5 \%)$ & \\
\hline Double lipomas & $1(2 \%)$ & $16(55 \%)$ & & \\
\hline
\end{tabular}

Values represent numbers of patients (\%) unless otherwise indicated.

tends laterally to the ventral side of the spinal cord, with the nerve roots inside, without forming a clear margin of fusion line. Finally, because the failed process extends into secondary neurulation, the conus medullaris is not recognized, and the lipoma often occupies the caudal end of the spinal canal.

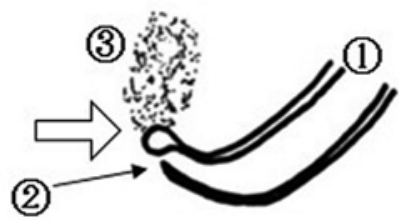

Closure of caudal neuropore and formation of caudal eminence

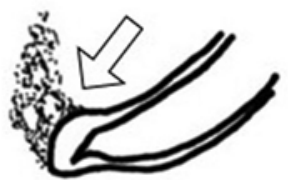

Cavitation and connection

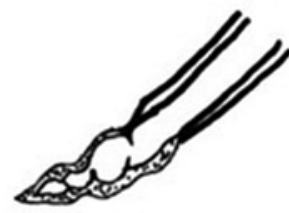

Fusion

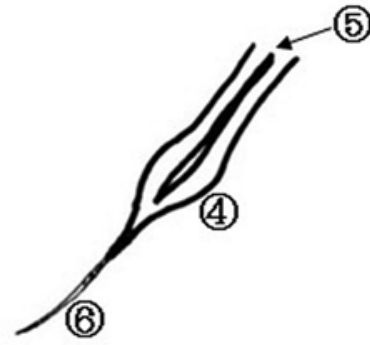

Regression

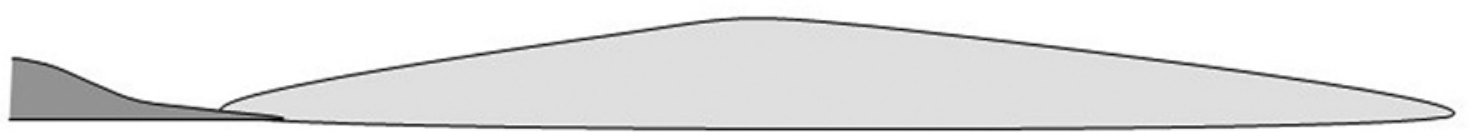

Primary neurulation

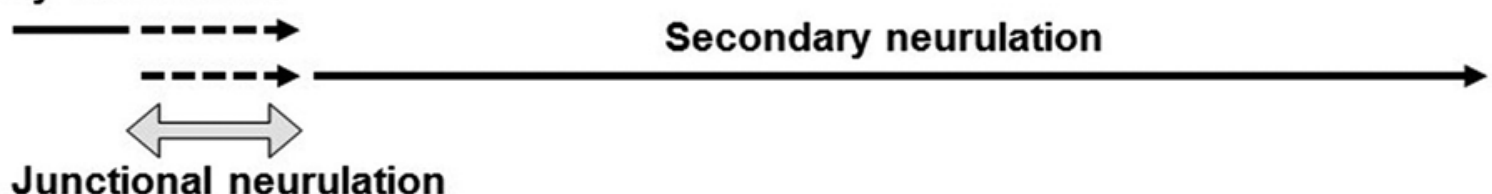

Separation of cloaca and organogenesis

Formation of external genitalia $=-=$

(1): Neural tube (2): caudal neuropore
(4): conus medullares (5): central canal
(6): filum teminence
: Junctional region

FIG. 7. Secondary neurulation (indicated by lighter shading). At the end of primary neurulation (indicated by darker shading), the caudal neuropore closes, and the caudal eminence forms distal to the neural tube. The caudal eminence undergoes cavitation and connects to the neural tube at the junctional region, fusing with the neural tube and regressing to form the filum terminale. When the transition from primary to secondary neurulation overlaps, junctional neurulation results. During the period of secondary neurulation, separation of the cloaca starts at postovulatory Week 5. Formation of the external genitalia begins at postovulatory Week 7. The simultaneity of these processes corresponds with the frequency of associated anomalies occurring in each type of spinal lipoma. 
Chapman's classification

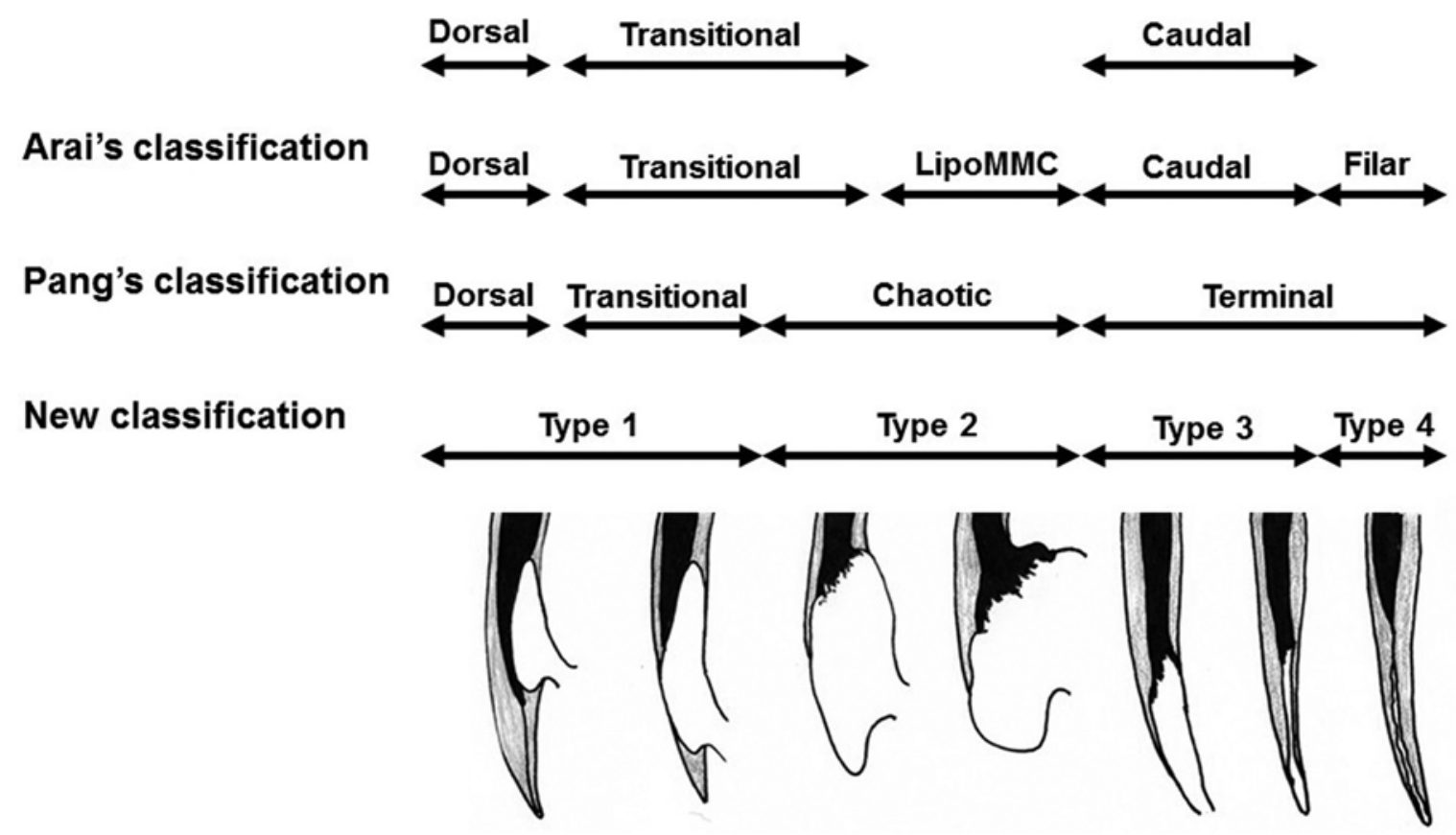

FIG. 8. Comparison of spinal lipoma taxonomies. The difference between the new and conventional classifications is shown. In Type 1 spinal lipomas, the conus medullaris is located caudally or ventrally with respect to the spinal lipoma. In Type 2, the conus medullaris is unformed or incompletely formed and fuses with the spinal lipoma without clear borderline. In Type 3, the conus medullaris is incompletely formed and fuses with the caudally located spinal lipoma. There is no dural defect on the dorsal side and no pathological spina bifida. In Type 4, the conus medullaris is present and moves toward the filum terminale, which forms the filum lipoma in its distal portion. LipoMMC = lipomyelomeningocele.

\section{Associated Anomalies and the New Spinal Lipoma Classification System}

The new spinal lipoma classification presented here correlates with the incidence of associated anomalies (Table 2). Separation and formation of anorectal and urogenital organs proceed after postovulatory Week 5 , simultaneously with secondary neurulation. Anorectal and/or sacral anomalies are associated with Type 2 and 3 spinal lipomas because they form simultaneously with the separation of the cloaca. Type 4 spinal lipoma showed less association with anorectal and/or sacral anomalies probably due to its formation at a late stage in secondary neurulation. This fact also accounts for the higher association of Type 4 spinal lipoma with urogenital anomalies because formation of external genitalia begins at postovulatory Week 7, corresponding to a late stage in secondary neurulation. Type 2 spinal lipoma also showed by far the highest frequency of association with the double lipoma. Type 2 spinal lipoma develops during the period extending from the end of primary neurulation to early secondary neurulation, including the so-called "junctional neurulation," potentially giving rise to more complex forms of spinal lipoma.

\section{Clinical and Surgical Implications}

The new classification based on the stages of embryogenesis corresponds well to the clinical and surgical findings (Fig. 9). Type 1 spinal lipomas usually present skin stigmata. The adipose tissue extends through the pathologi- cal spinal bifida and the fascial and dural defects to merge with the intradural lipoma. Diagnosis is made around the neonatal period. The spinal lipoma is located dorsal or dorsolateral to the spinal cord and compresses the cord ventrally. Surgical intervention is moderately difficult, but radical resection is possible since the fusion line exists. ${ }^{15,16,18,20}$ Since Type 1 spinal lipoma develops only within a narrow time frame at the end of primary neurulation, association with other anomalies is rare, as can be seen in Table 2.

Type 2 spinal lipomas have a similar appearance as Type 1. Diagnosis of Type 2 lipomas is also possible in the neonatal period. Extension of the lipoma to the lateral or ventral side is not unusual and often involves neural elements within the lipoma. Association with other anomalies occurs more frequently than in other types of spinal lipoma. Interestingly, because its development occurs in the period extending from primary to secondary neurulation, the frequency of double lipomas was the highest for this type (as shown in Table 2). Surgical removal tends to be technically demanding. The presence of a double lipoma needs to be considered, and exploration of the distal end of the spinal canal is strongly recommended. Surgery for Type 2 spinal lipoma poses a challenge to pediatric neurosurgeons. Radical resection is not indicated. ${ }^{15,16,18,20}$

Type 3 spinal lipoma has little or no skin stigmata. Physiological spina bifida is present, but there is no pathological spina bifida. The dorsal aspect of the lumbosacral fascia and dura have no defect. The dural defect is present at the caudal end, where subcutaneous fat invades the 
Type 1 spinal lipoma: Pure primary neurulation failure
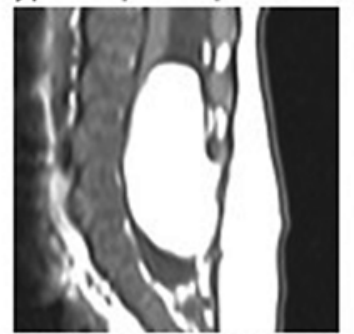

Clinical findings
Spina bifida
Timing of diagnosis
Relationship with conus medullaris
Surgical implications
Assoclated anomalies

Skin stigmata; fascial and dural defect

Pathological spina bifida

Neonatal penod

Dorsal location predominant with some uneven lateral extension

Not easy, but not extremely difficult; radical resection possible

Usually none

\section{Type 2 spinal lipoma: Ranging from primary to secondary neurulation failure}

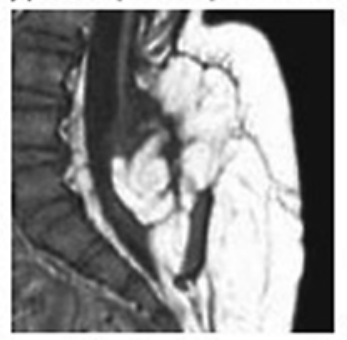

\begin{tabular}{|l|l|}
\hline Clinical findings & Skin stigmata; fascial and dural defect \\
Spina bifida & Pathological spina bifida \\
Timing of diagnosis & Neonatal period
\end{tabular}

Timing of diagnosis

Relationship with conus medullaris

Dorsal to lateral, partly ventral extension with neural

Surgical implications

Associated anomalles

elements Inside not unusual

Difficult; radical resection impossible

Anorectal/sacral anomalles can be seen

Type 3 spinal lipoma: Secondary neurulation failure (early phase)

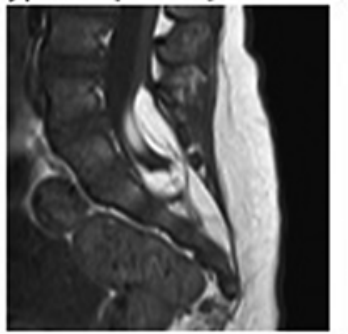

\begin{tabular}{|l|l|}
\hline $\begin{array}{l}\text { Clinical findings } \\
\text { Spina bifida } \\
\text { Timing of diagnosis }\end{array}$ & $\begin{array}{l}\text { No or only tiny skin stigmata; no dorsal dural defect } \\
\text { Physiological spina bifida } \\
\text { Late infancy or school age (not unusual) } \\
\text { Relationship with conus medullaris }\end{array}$ \\
$\begin{array}{l}\text { Caudal to conus medullaris, but without clear borderline; } \\
\text { Lateral, ventral extension not unusual } \\
\text { Surgical implications }\end{array}$ & $\begin{array}{l}\text { Moderately difficult in some cases; radical resection not } \\
\text { indicated } \\
\text { Anorectal/sacral anomalles often seen }\end{array}$ \\
\hline Assoclated anomalles &
\end{tabular}

Type 4 spinal lipoma: Secondary neurulation failure (late phase)

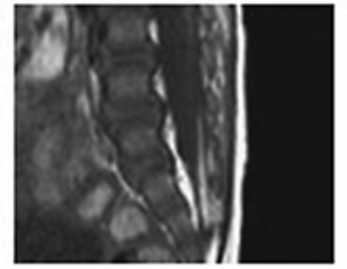

Clinical findings
Spina bifida
Timing of diagnosis
Relationship with conus medullaris
Surgical implications
Associated anomalies

Tiny skin stigmata (dimple)
Physiological spina bifida
Intancy
Normal conus medullaris, lipoma of the filum terminale
Simple and usually straightforward
Anorectal/urogenital anomalles not unusual

FIG. 9. Clinical features of each type of spinal lipoma. Clinical features of Type 1 through Type 4 spinal lipomas are summarized with representative T1-weighted sagittal MR images.

spinal canal through an enlarged sacral hiatus. Diagnosis can sometimes be delayed when a tiny skin stigma is overlooked after birth, a fact possibly accounting for the higher median age at surgery of patients with Type 3 spinal lipomas than of those with other types (Table 2). Association of various anomalies is often observed. It is not unusual for the lipoma to be found during detailed examination for associated anomalies in the neonatal period and infancy. ${ }^{9}$ Symptoms due to neurogenic bladder often lead to diagnosis in toddlers and school-age children. The lipoma attaches to the conus medullaris distal to the caudalmost nerve roots. Surgery is not difficult, but the adhesion of nerve roots to the surface of the lipoma sometimes poses a challenge. No radical resection is recommended unless a retained medullary cord is present between the lipoma and conus medullaris. ${ }^{17}$

Type 4 spinal lipoma is nearly synonymous with the filum lipoma. A tiny dimple in the sacrococcygeal region often provides a clue for diagnosis, although its pathological significance is still disputed. ${ }^{5,6}$ Surgery, if indicated, is straightforward in the majority of cases. On these grounds, Type 4 spinal lipoma is considered distinct from Type 3 in our classification, although both are considered to be instances of "terminal lipoma" in Pang's classification..$^{14,20}$

\section{Conclusions}

The authors propose a new classification of spinal lipomas based on the stages of embryogenesis, which has the 2-fold merit of explaining spinal lipoma formation more seamlessly than previously published classificatory systems while demonstrating a practical correspondence to the clinical features and surgical difficulty of for each type.

Newly accumulated knowledge regarding development of the caudal neural tube is the key to understanding the new classification. The new concept of junctional 
neurulation bridging primary and secondary neurulation can explain the development of complex forms of spinal lipoma, such as the chaotic spinal lipoma in Pang's classification. ${ }^{4,16,20}$ The formation of a spinal lipoma can be conceived as a continuous process crossing the juncture between primary and secondary neurulation in some cases.

The new classification proposed here is still preliminary, and other subgroups may well exist within each type of spinal lipoma described above. The advantage of the new classification resides in its use of the embryonic stages of caudal neural tube formation, which correlates well with the clinical and surgical findings of spinal lipomas for each type. Further study and verification are necessary to establish the clinical utility of the proposed system.

\section{Acknowledgments}

We thank Mr. James Robert Valera for his editorial assistance and professional advice on the manuscript.

\section{References}

1. Arai H, Sato K, Okuda O, Miyajima M, Hishii M, Nakanishi $\mathrm{H}$, et al: Surgical experience of 120 patients with lumbosacral lipomas. Acta Neurochir (Wien) 143:857-864, 2001

2. Chapman PH: Congenital intraspinal lipomas: anatomic considerations and surgical treatment. Childs Brain 9:37-47, 1982

3. Copp AJ, Greene NDE: Neural tube defects-disorders of neurulation and related embryonic processes. Wiley Interdiscip Rev Dev Biol 2:213-227, 2013

4. Dady A, Havis E, Escriou V, Catala M, Duband JL: Junctional neurulation: a unique developmental program shaping a discrete region of the spinal cord highly susceptible to neural tube defects. J Neurosci 34:13208-13221, 2014

5. Gomi A, Oguma H, Furukawa R: Sacrococcygeal dimple: new classification and relationship with spinal lesions. Childs Nerv Syst 29:1641-1645, 2013

6. Harada A, Nishiyama K, Yoshimura J, Sano M, Fujii Y: Intraspinal lesions associated with sacrococcygeal dimples. J Neurosurg Pediatr 14:81-86, 2014

7. McLone DG, Naidichi TP: The tethered spinal cord, in McLaurin RL, Shut L, Venes JL, et al (eds): Pediatric Neurosurgery, ed 2. Philadelphia: Saunders, 1989, pp 71-75

8. McLone DG, Thompson DNP: Lipomas of the spine, in McLone DG (ed): Pediatric Neurosurgery: Surgery of the Developing Nervous System. Philadelphia: Saunders, 2001, pp 289-301

9. Morimoto K, Takemoto O, Wakayama A: Tethered cord associated with anorectal malformation. Pediatr Neurosurg 38:79-82, 2003

10. Müller F, O'Rahilly R: The development of the human brain, the closure of the caudal neuropore, and the beginning of secondary neurulation at stage 12. Anat Embryol (Berl) 176:413-430, 1987

11. Müller F, O'Rahilly R: The primitive streak, the caudal eminence and related structures in staged human embryos. Cells Tissues Organs 177:2-20, 2004

12. Nievelstein RAJ, Hartwig NG, Vermeij-Keers C, Valk J: Embryonic development of the mammalian caudal neural tube. Teratology 48:21-31, 1993

13. O'Rahilly R, Müller F: Neurulation in the normal human embryo. Ciba Found Symp 181:70-89, 1994

14. Pang D: Caudal agenesis and dysembryogenesis of the caudal spinal cord, in Pang D (ed): Disorders of the Pediatric Spine. New York: Raven Press, 1995, pp 277-308

15. Pang D: Spinal cord lipomas, in Pang D (ed): Disorders of the Pediatric Spine. New York: Raven Press, 1995, pp 175-201

16. Pang D: Total resection of complex spinal cord lipomas: how, why, and when to operate? Neurol Med Chir (Tokyo) 55:695-721, 2015

17. Pang D, Zovickian J, Moes GS: Retained medullary cord in humans: late arrest of secondary neurulation. Neurosurgery 68:1500-1519, 2011

18. Pang D, Zovickian J, Oviedo A: Long-term outcome of total and near-total resection of spinal cord lipomas and radical reconstruction of the neural placode: part I-surgical technique. Neurosurgery 65:511-529, 2009

19. Pang D, Zovickian J, Oviedo A: Long-term outcome of total and near-total resection of spinal cord lipomas and radical reconstruction of the neural placode, part II: outcome analysis and preoperative profiling. Neurosurgery 66:253-273, 2010

20. Pang D, Zovickian J, Wong ST, Hou YJ, Moes GS: Surgical treatment of complex spinal cord lipomas. Childs Nerv Syst 29:1485-1513, 2013

21. Rizk E, Adeeb N, Hussein AE, Tubbs RS, Rozzelle CJ, Oakes WJ: Duplicated filum terminale in the absence of split cord malformation: a potential cause of failed detethering procedures. Childs Nerv Syst 30:709-711, 2014

22. Sadler TW: Digestive system, in Langman's Medical Embryology, ed 13. Philadelphia: Wolters Kluwer, 2015, pp 225-249

23. Sadler TW: Urogenital system, in Langman's Medical Embryology, ed 13. Philadelphia: Wolters Kluwer, 2015, pp 250-277

24. Saitsu H, Yamada S, Uwabe C, Ishibashi M, Shiota K: Development of the posterior neural tube in human embryos. Anat Embryol (Berl) 209:107-117, 2004

25. Shimokita E, Takahashi Y: Secondary neurulation: Fatemapping and gene manipulation of the neural tube in tail bud. Dev Growth Differ 53:401-410, 2011

26. Tokunaga S, Morioka T, Hashiguchi K, Samura K, Yoshida F, Miyagi Y, et al: Double lumbosacral lipomas of the dorsal and filar types associated with OEIS complex: case report. Neurol Med Chir (Tokyo) 49:487-490, 2009

27. Van Allen MI, Kalousek DK, Chernoff GF, Juriloff D, Harris M, McGillivray BC, et al: Evidence for multi-site closure of the neural tube in humans. Am J Med Genet 47:723-743, 1993

28. Yoshifuji K, Morota N, Ihara S: [Developmental feature of the lumbosacral vertebral arch in childhood.] Shoni No Noshinkei 32:426-429, 2007 (Jpn)

\section{Disclosures}

The authors report no conflict of interest concerning the materials or methods used in this study or the findings specified in this paper.

\section{Author Contributions}

Conception and design: Morota. Acquisition of data: all authors. Analysis and interpretation of data: Morota. Drafting the article: Morota. Critically revising the article: Morota, Ogiwara. Reviewed submitted version of manuscript: Morota, Ihara. Approved the final version of the manuscript on behalf of all authors: Morota. Statistical analysis: Morota. Administrative/technical/material support: Morota. Study supervision: Morota.

\section{Correspondence}

Nobuhito Morota, Tokyo Metropolitan Children's Medical Center, Division of Neurosurgery, 2-8-29, Musashi-dai, Fuchu, Tokyo,

Japan 183-8561. email: nobu.m01@gmail.com. 